\title{
Tellurite glass microcavity resonators integrated on a silicon photonics platform
}

\author{
Henry C. Frankis, Dawson B. Bonneville, and Jonathan D. B. Bradley* \\ McMaster University, Department of Engineering Physics, Hamilton, Ontario, Canada
}

\begin{abstract}
We report on the design and measurement of tellurium oxide microcavity resonators coupled to silicon bus waveguides on silicon photonic chips. The resonators are fabricated using a standard silicon photonics foundry processing flow in which the $\mathrm{SiO}_{2}$ top-cladding is etched in a ring shape and aligned next to a silicon bus waveguide. The resulting microtrench is coated in a tellurium oxide film by reactive sputtering in a post-processing step to form the waveguiding layer of the resonator. A $100-\mu$ m radius trench with a $1115-\mathrm{nm}$-thick $\mathrm{TeO}_{2}$ film is measured to have an internal $Q$ factor of $0.9 \times 10^{5}$. Smoothing the etch wall surface with a fluoropolymer coating is shown to enhance the $Q$ factor of several devices, with a trench coated in a 630-nm-thick $\mathrm{TeO}_{2}$ film demonstrating a $Q$ factor of $2.1 \times 10^{5}$ corresponding to $1.7-\mathrm{dB} / \mathrm{cm}$ waveguide loss. These results demonstrate a potential pathway toward monolithic integration of tellurite glass-based nonlinear and rare-earth-doped devices compatible with silicon photonics platforms. () The Authors. Published by SPIE under a Creative Commons Attribution 4.0 Unported License. Distribution or reproduction of this work in whole or in part requires full attribution of the original publication, including its DOI. [DOI: 10.1117/1.JOM.1.2.024002]
\end{abstract}

Keywords: silicon photonics; tellurium oxide; optical microcavities.

Paper 20019 received Nov. 19, 2020; accepted for publication Feb. 1, 2021; published online Mar. 9, 2021.

\section{Introduction}

Integrated photonic circuits based in silicon-on-insulator (SOI) waveguides are a leading photonics platform due to their mature complementary metal-oxide-semiconductor (CMOS) compatible fabrication technology that can leverage the existing silicon electronics fabrication infrastructure. ${ }^{1,2}$ A large library of established passive silicon photonic components, such as fiber-chip couplers, wavelength-division-multiplexors, and filters are well developed, as well as opto-electronic devices, such as modulators and photodetectors. ${ }^{2-4}$ However, challenges still exist in fabricating light sources with silicon waveguides, including optically pumped nonlinear and rare-earth-doped active photonic devices, due to silicon's large two-photon absorption at telecommunications wavelengths ${ }^{5,6}$ and low rare earth solubility, ${ }^{7,8}$ respectively.

In contrast, silica and other glass-based resonators have demonstrated efficient optically pumped devices, including frequency combs, ${ }^{9,10}$ Raman lasers, ${ }^{11,12}$ and rare earth lasers. ${ }^{13-15}$ High $Q$ factor glass resonators typically take the form of either microspheres/bubbles, ${ }^{16,17}$ formed by melting the tip of a glass fiber, or microtoroids/disks, ${ }^{18}$ fabricated by etching and then smoothing a toroidal disk on an oxidized silicon wafer by laser irradiation, among other similar resonator designs. The highly smooth surfaces of these devices allow them to reach ultrahigh $Q$ factors of $>10^{8}$, but because they are free standing structures, they must be coupled directly to optical fibers. Additionally, although some demonstrations have been made, ${ }^{19,20}$ opto-electronic functionality in glass-based systems is difficult to achieve. Techniques to fabricate glass-based resonator structures monolithically compatible with silicon photonic systems would merge the complimentary advantages of both platforms.

Integration of silica resonators directly with both silicon ${ }^{21,22}$ and silicon nitride ${ }^{23}$ bus waveguides has been demonstrated by locally etching the silicon substrate underneath the buried oxide (BOX) layer to create a suspended silica layer that acts as the waveguiding layer of the resonator. Although high $Q$-factor $\left(>10^{6}\right)$ devices have been demonstrated in these platforms,

*Address all correspondence to Jonathan D. B. Bradley, frankihc@mcmaster.ca 
many additional fabrication steps that are nonstandard processes for silicon photonic foundries, such as etching the substrate, are required.

A simpler approach to integration of glass-based resonators uses a microtrench design, whereby the top cladding of a foundry fabricated photonic chip is etched in the shape of a circular cavity and filled with a waveguiding glass layer in post-processing. However, to maintain appropriate levels of index contrast between the resonator and the silica cladding, glasses with a larger refractive index than that of silica must be used. Aluminum oxide glass cavities fabricated in this manner and aligned to silicon nitride bus waveguides have demonstrated high $Q$ factors of greater than $10^{6},{ }^{24}$ with demonstrations of rare earth lasing with erbium ${ }^{25}$ and thulium dopants ${ }^{26}$ and four wave mixing. ${ }^{27}$ Advanced multilayer foundry processes can be used to vertically couple light from silicon nitride to silicon waveguides to combine these devices with electro-optic functionality, ${ }^{28-30}$ but for lower cost fabrication technology, it would be preferable to be able to couple the resonator directly to the silicon waveguide layer. Although aluminum oxide resonators have been highly successful in combination with silicon nitride waveguides, it is much more difficult to phase match the low refractive index of aluminum oxide (1.65) to a silicon bus waveguide, making alternative glasses more preferable for direct silicon integration. Recently, a chalcogenide resonator formed in a microtrench and smoothed with a dewetting process and coupled to a silicon waveguide with $Q$ factors of $6.0 \times 10^{5}$ was demonstrated. ${ }^{31}$ These resonators are highly promising for mid-infrared devices, but low thermal and photostability in chalcogenide glasses are a challenge. ${ }^{32-34}$

In this work, we use a tellurium oxide glass coating to form microtrench resonators directly coupled to SOI bus waveguides fabricated in a standard silicon photonics foundry. Tellurite glass's large refractive index $(2.08$ at $1550 \mathrm{~nm})$ and demonstrated low optical propagation losses $^{35,36}$ make it a suitable candidate for fabricating high $Q$ resonators compatible with a silicon photonics platform. Additionally, tellurite glass has a large nonlinear coefficient, ${ }^{37,38}$ large Raman gain, ${ }^{39}$ and has been used as a host for erbium- ${ }^{40-42}$ and thulium ${ }^{43}$-doped waveguide amplifiers and lasers. We have previously shown applications of this platform toward thermal and evanescent waveguide sensor devices. ${ }^{44}$ Here, we provide greater detail on the cavity design and device properties, as well as improved device performance, showing $Q$ factors of up to $2.1 \times$ $10^{5}$ by using polymer coatings to smooth the microtrench sidewall. These results demonstrate a platform for future integration of nonlinear photonic and rare-earth-doped active tellurite resonators on a silicon photonics platform, for applications including Kerr comb sources for sensing and spectroscopy ${ }^{45}$ laser biosensors for diagnostic systems on a chip, ${ }^{46}$ and high-power light sources for communications and detection and ranging. ${ }^{47}$

\section{Tellurium Oxide Microresonator Fabrication}

The silicon chips were fabricated in the Institute of Microelectronics/Advanced Micro Foundry as part of an active silicon photonics multiproject wafer run. The silicon waveguides were fabricated on a $2-\mu \mathrm{m}$ BOX layer with a standard $220-\mathrm{nm}$-thick silicon layer. The foundry offers three waveguide etch steps, allowing for fabrication of strip and or rib waveguides, patterned using 193-nm-deep ultraviolet lithography. Following waveguide patterning, the wafer undergoes several processing steps for the fabrication of active devices, including: boron and phosphorous implants, germanium epitaxy, and metal contact vias. Two rows of metal interconnect layers were deposited, and a total thickness of 3- $\mu \mathrm{m} \mathrm{SiO}{ }_{2}$ top-cladding was deposited above the BOX. Following the final growth of the top cladding, windows were defined using a timed etch, designed to etch down to the top of the BOX layer, in a process typically used to expose waveguides for sensor devices. For the resonator design, the window etch step was applied over a region with no underlying waveguide in the shape of a ring resonator with a $10-\mu \mathrm{m}$-wide trench and an outer diameter of $100 \mu \mathrm{m}$, aligned next to a 220-nm-thick silicon strip waveguide. Nominal gaps between the interior waveguide and exterior cavity wall ranging from $-0.3 \mu \mathrm{m}$ within the cavity wall to account for the lateral offset that occurs due to the sloped oxide etch wall to $0.3 \mu \mathrm{m}$ past the cavity wall were used with an etch-mask alignment accuracy of $\pm 10 \mathrm{~nm}$. In the coupling region, the typically $0.5-\mu \mathrm{m}$-wide waveguide is reduced to a width of $0.3 \mu \mathrm{m}$, which both increases the size of the waveguide mode's evanescent tail and better 
matches the effective index of the microcavity mode. We also designed the waveguides to be pulley coupled around the resonator over $60 \mathrm{deg}$, creating a 104- $\mu \mathrm{m}$ effective coupling length and increasing the coupling coefficient between the bus waveguide and cavity. The SOI wafer then underwent a deep etch process along the edges of the chips to create smooth facets for edge coupling after which the chips were diced and transferred from the foundry.

Following SOI chip fabrication in the foundry, we deposited a $\mathrm{TeO}_{2}$ coating layer using a straightforward low-temperature post-processing step. We deposit the $\mathrm{TeO}_{2}$ coating using reactive sputtering, whereby a high-purity metallic tellurium target is sputtered in an argon/oxygen environment and the sputtered tellurium atoms react with the oxygen ambient to form a tellurium oxide layer on the samples. The reactive sputtering process allows for films to be deposited on either individual chips or on a wafer scale, with high deposition rates and high film quality. The deposition process is carried out at ambient temperature, enabling integration with metal layers and active silicon photonic devices. Details on the parameters of the sputtering process can be found in our previous publication. ${ }^{36}$ The $\mathrm{TeO}_{2}$ layer can be doped with rare earth ions by simultaneously sputtering from a rare earth target on an additional sputtering gun within the system during film deposition. ${ }^{42,43}$ In this demonstration, we coat the entirety of the chip as no opto-electronic functionality is used, although in the future, a lift off or shadow masking process ${ }^{48}$ could be used around the microcavity to prevent tellurite glass coating over metal contacts. An overview of the full fabrication process used in this design can be seen in Fig. 1(a), with scanning electron microscope (SEM) images of the fabricated device displayed in Figs. 1(b) and 1(c).

As seen in the cross-sectional SEM image of the cavity after a focused-ion-beam (FIB) cut in Fig. 1(c), the silica top-cladding etch extends significantly below the level of the silicon waveguide and the top of the BOX by approximately $1 \mu \mathrm{m}$ based on the image, creating a vertical offset between the silicon bus waveguide and the microtrench cavity. A second design run from

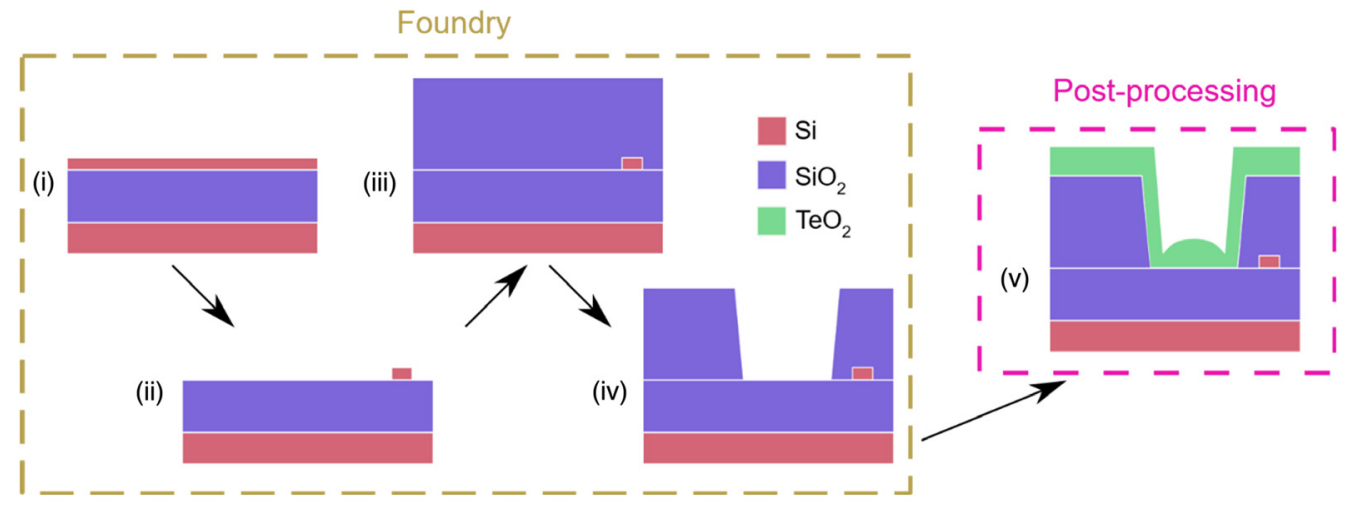

(a)

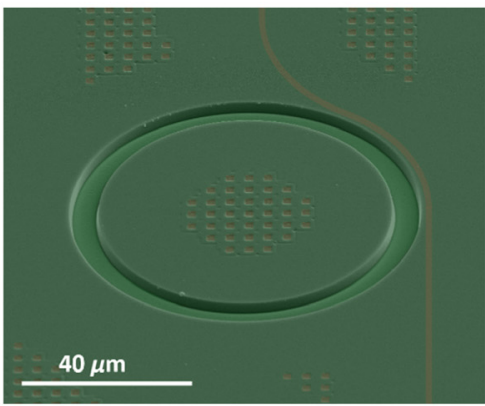

(b)

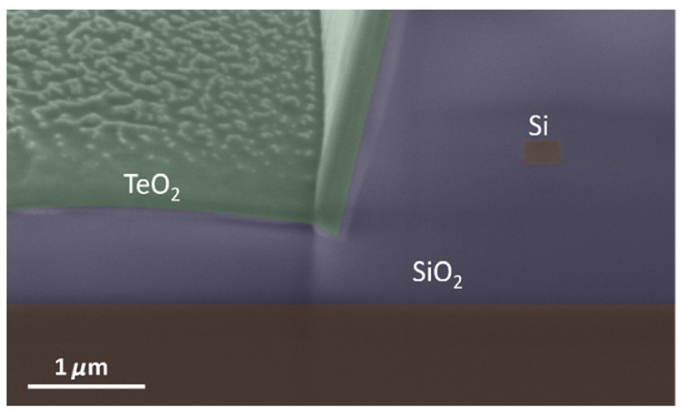

(c)

Fig. 1 (a) Fabrication process of the tellurium oxide resonator: (i) fabrication is carried out on a SOI wafer including a 220-nm-thick silicon layer on a 2- $\mu \mathrm{m} \mathrm{BOX,} \mathrm{(ii)} \mathrm{the} \mathrm{silicon} \mathrm{layer} \mathrm{is} \mathrm{patterned}$ into a bus waveguide, (iii) a $3-\mu \mathrm{m}$ silicon dioxide top cladding is deposited, (iv) the top-cladding layer is etched to pattern a circular microtrench, and (v) post-processing tellurium oxide deposition into the microtrench, forming the resonator layer. SEM of a microcavity with a $400-\mathrm{nm}$-thick $\mathrm{TeO}_{2}$ layer from a (b) top view and (c) cross-sectional profile after an FIB cut. 
the foundry was used in an attempt to reduce the magnitude of the overetch, where a $100 \times$ $100 \mu \mathrm{m}^{2}$ square oxide etch window test structure was added to be able more precisely quantify the magnitude of the overetch for the chips using surface profilometry. The overetch of several samples are measured to be $245 \mathrm{~nm}$ on average with a standard deviation of $85 \mathrm{~nm}$, demonstrating a less significant overetch compared to the initial run. From Fig. 1(c), it can be seen that the etch depth is approximately $0.3 \mu \mathrm{m}$ deeper near the cavity sidewall, where the optical mode is confined, as compared to the center of the cavity. As the surface profilometer has a $10-\mu \mathrm{m}$ diameter probe tip, it is unable to resolve the etch depth near the sidewall of the etch, instead measuring closer to the center of the cavity. Based on this, we estimate that the chips studied here from the second design run have an average overetch of approximately $0.5 \mu \mathrm{m}$ near the cavity sidewall. In addition to the vertical offset from the overetch, the angled window etch creates a lateral offset of the cavity from the designed nominal gap. The estimated 80-deg etch angle creates a lateral offset of approximately $0.6 \mu \mathrm{m}$ versus the designed window-waveguide gap. Considering the lateral and vertical offsets, a nominal waveguide-to-cavity coupling gap of $0.2 \mu \mathrm{m}$ becomes an effective coupling gap of approximately $0.8 \mu \mathrm{m}$, significantly reducing the coupling coefficient.

\section{Simulated Microresonator Properties}

We used finite element eigenmode simulations based on the cavity cross section to analyze the properties of the microcavity devices. Figure 2(a) shows the model of the cavity cross section, and the relevant device dimensions used in the simulations. The foundry process and etch mask design determine the dimensions of the silicon waveguide $\left(t_{\mathrm{Si}}=0.22 \mu \mathrm{m}\right.$ and $\left.w_{\mathrm{Si}}=0.30 \mu \mathrm{m}\right)$, the radius of the microcavity, $R$, the thickness of the BOX separating the bottom of the microtrench from the silicon substrate, $t_{\mathrm{BOX}}$, the trench sidewall angle, and the nominal waveguide to microcavity coupling gap, $g$, while the thickness of the $\mathrm{TeO}_{2}$ coating, $t_{\mathrm{TeO} 2}$, is controlled by the length of the $\mathrm{TeO}_{2}$ deposition performed in post-processing. The use of a confocal sputtering gun arrangement and rotating substrate holder in the $\mathrm{TeO}_{2}$ deposition process is assumed to result in a deposition profile within the cavity similar to that reported in Ref. 24 for aluminum oxide cavities. The $\mathrm{TeO}_{2}$ film coats the surface of the chip and the bottom of the microtrench structure. Near the cavity sidewall, approximately half the deposited thickness, $t_{\mathrm{TeO} 2} / 2$, is expected to be grown along the sidewall while the $\mathrm{TeO}_{2}$ layer along the bottom of the cavity thins into the corner where it meets the sidewall. We estimate the shape using an ellipse, with a minor (vertical) axis of half the deposited film thickness aligned to the bottom of the trench, and a major (horizontal) axis aligned to the bottom corner of the trench. For all simulations, we assume a major axis width, $w_{\text {corner }}$, of $2 \mu \mathrm{m}$, as little variation is seen in simulation results with major axis widths varying from 0.5 to $4 \mu \mathrm{m}$. The waveguide bend created by the radius of

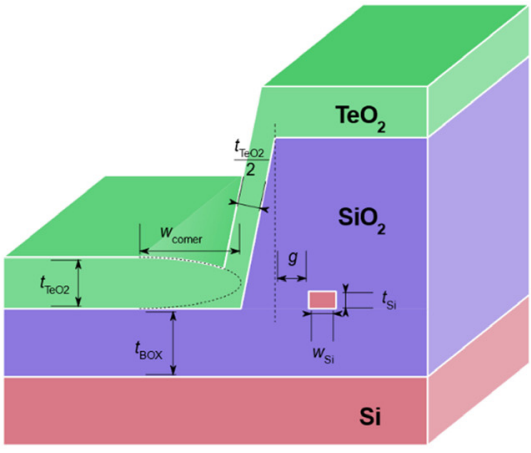

(a)

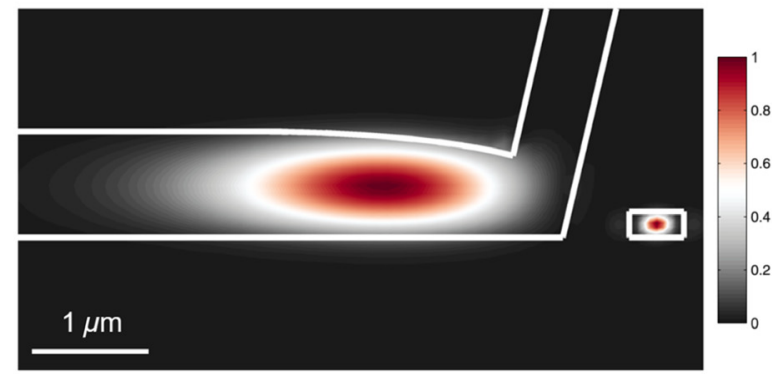

(b)

Fig. 2 (a) $\mathrm{TeO}_{2}$ cavity cross-sectional profile and relevant device dimensions used in simulations. (b) Simulated TE-polarized electric-field profile for the fundamental mode of a $\mathrm{TeO}_{2}$ cavity with a $900-\mathrm{nm}$-thick $\mathrm{TeO}_{2}$ coating and a $100-\mu \mathrm{m}$ bend radius, next to the mode profile of a silicon bus waveguide with a $0.22-\mu \mathrm{m}$ height and $0.30-\mu \mathrm{m}$ width at $1550-\mathrm{nm}$ wavelength, each normalized to their respective peak electric-field intensity. 
the cavity pushes the optical mode within the tellurite glass layer toward the outer bottom corner of the trench. For these simulations, we assumed a flat trench bottom for simplicity. A sample simulated optical mode profile of a resonator with a $900-\mathrm{nm}$-thick $\mathrm{TeO}_{2}$ coating, at a $1550-\mathrm{nm}$ wavelength with transverse-electric (TE) polarization next to a sample mode of the silicon bus waveguide, with a $0.30-\mu \mathrm{m}$ width $\left(w_{\mathrm{Si}}\right)$ and $0.22-\mu \mathrm{m}$ thickness $\left(t_{\mathrm{Si}}\right)$, at the same wavelength and polarization state, can be seen in Fig. 2(b).

To allow for the fabrication of effective nonlinear and rare-earth-doped microcavity devices, a primary requirement is low waveguide loss or, equivalently, high $Q$ factor. The microcavity $Q$ factor is influenced by losses due to scattering at rough interfaces, material-related absorption, bend-related radiation losses, and mode leakage into the silicon substrate. Roughening of the silica interface from the etch process can introduce scattering losses into the cavity, and the tellurite glass films can also exhibit absorption and scattering losses, although they should be minimal for high optical quality deposition processes. Radiation losses can be effectively minimized in the device by appropriately designing the cavity to limit radiation pathways associated with bending and substrate leakage. We calculated the bending radiation limited $Q$ factor of the cavity with $\mathrm{TeO}_{2}$ film thicknesses ranging from 500 to $1100 \mathrm{~nm}$ and microcavity outer bending radii ranging from 5 to $200 \mu \mathrm{m}$, and an infinitely thick BOX layer, assuming that bending radiation is the only source of loss in the cavity. The calculated loss was converted to an internal $Q$ factor ${ }^{49}$ and the results are shown in Fig. 3(a). The data show that thinner $\mathrm{TeO}_{2}$ films can allow for smaller bending radii before the radiation loss becomes significant, however, even devices with 1100-nm-thick $\mathrm{TeO}_{2}$ coatings are able to maintain bending radiation limited $Q$ factors of $>10^{9}$ at radii of $40 \mu \mathrm{m}$ and above. We also calculated the substrate radiation limited $Q$ factor versus the BOX thickness, $t_{\mathrm{BOX}}$, as shown in Fig. 3(b) for a fixed microcavity radius of $40 \mu \mathrm{m}$. We observe that a $2-\mu \mathrm{m} \mathrm{BOX}$ is sufficiently thick to limit substrate radiation to negligible values for all investigated $\mathrm{TeO}_{2}$ film thicknesses. However, as discussed earlier, the overetched microtrench structure results in an effective $t_{\mathrm{BOX}}$ of $1.5 \mu \mathrm{m}$ below the cavity on average. At BOX thickness of this magnitude, the substrate radiation losses become much more significant and are estimated to have substrate radiation limited $Q$ factors of $>10^{8}$, but further overetching as seen in the initial run of chips can limit $Q$ factors to potentially as low as $\sim 10^{5}$ to $10^{6}$ for a $t_{\mathrm{BOX}}$ of $1.0 \mu \mathrm{m}$. Therefore, to allow for higher $Q$ factor devices in the future, it is essential to ensure that the $\mathrm{SiO}_{2}$ layer thickness under the cavity is sufficiently large. Simulations with larger microcavity bending radii predict a minor decrease in substrate radiation limited $Q$ factors, as the mode becomes less strongly confined in the corner of the cavity.

To efficiently couple light to the $\mathrm{TeO}_{2}$ microcavity, the $\mathrm{Si}$ bus waveguide and cavity must be closely phase matched with similar effective indices. We calculated the effective index of a 220-nm-thick strip silicon waveguide for different waveguide widths to determine an appropriate

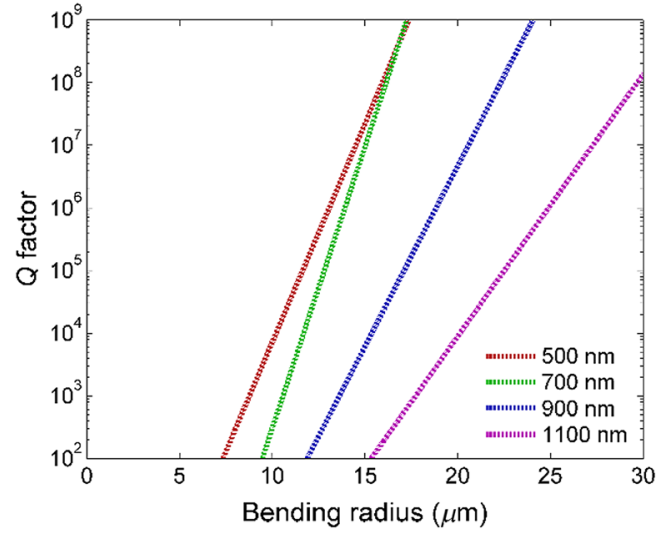

(a)

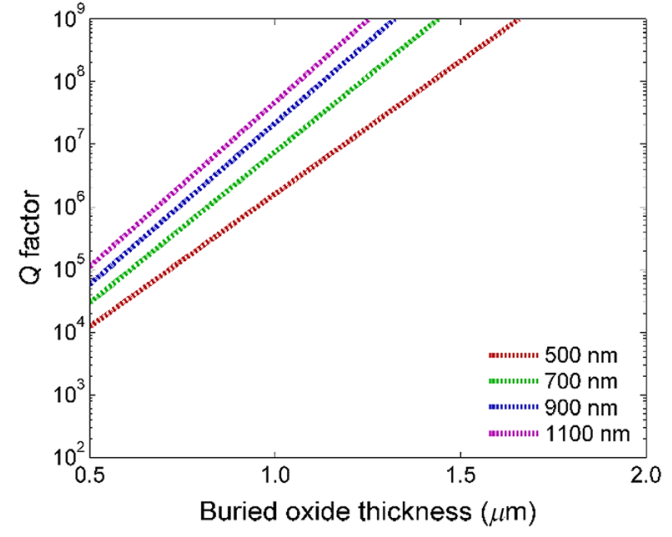

(b)

Fig. 3 Calculated internal $Q$ factor of $\mathrm{TeO}_{2}$ microcavities with 500-, 700-, 900-, and 1100-nm-thick $\mathrm{TeO}_{2}$ coatings limited by (a) bending radiation loss (for varying bend radius and an infinite $\mathrm{BOX}$ thickness) and (b) substrate radiation loss (for varying BOX thickness and fixed bend radius of $40 \mu \mathrm{m})$. 


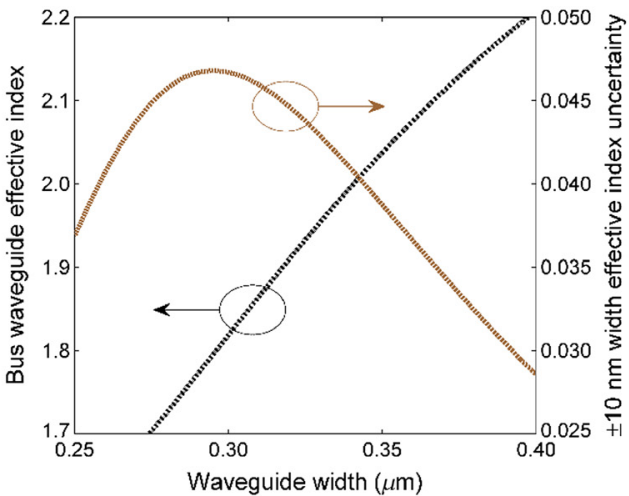

(a)

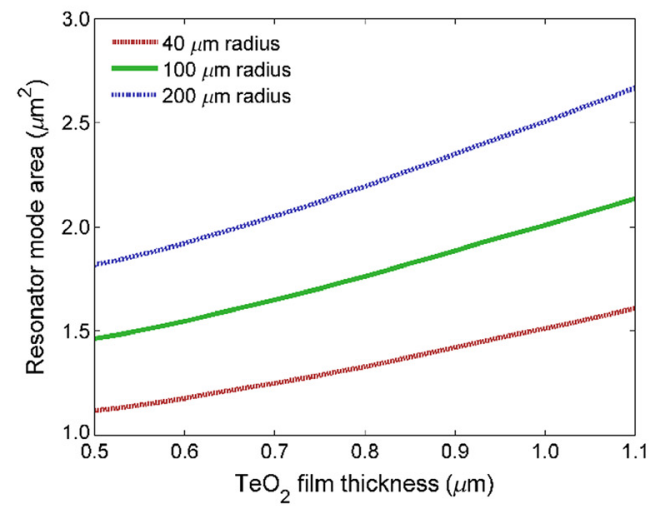

(c)

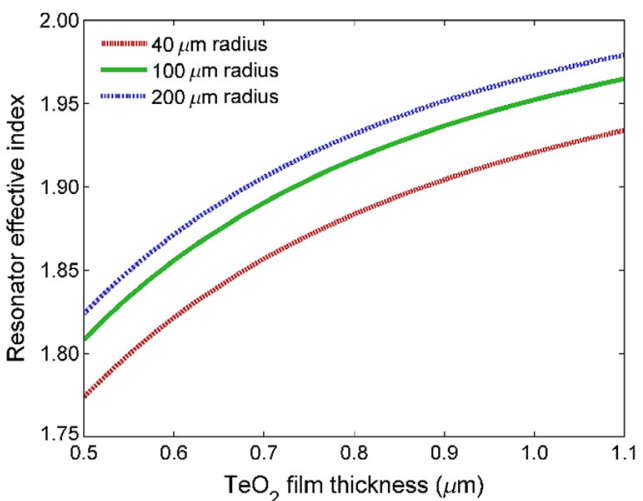

(b)

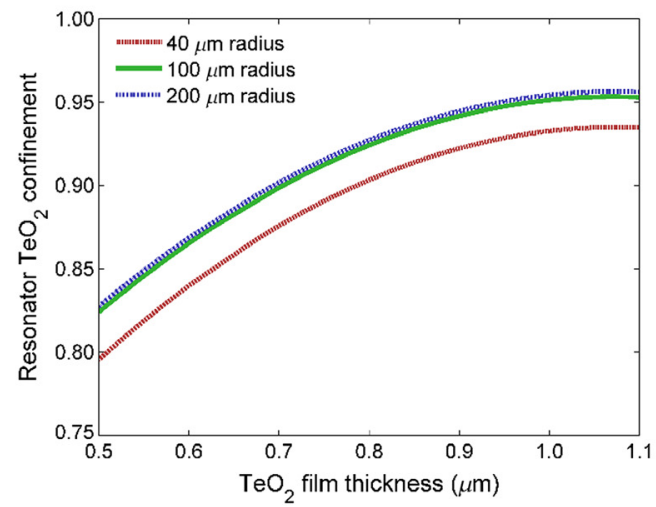

(d)

Fig. 4 Calculated (a) silicon bus waveguide effective index versus waveguide width, and effective index variation for $\mathrm{a} \pm 10$-nm waveguide width variation, and tellurite glass microcavity (b) effective index, (c) effective mode area, and (d) confinement factor within tellurium oxide versus deposited $\mathrm{TeO}_{2}$ film thickness for 40-, 100-, and $200-\mu \mathrm{m}$ radius microcavities.

bus width for coupling light to the resonator, as shown in Fig. 4(a). Thinner waveguide widths lead to expansion of the evanescent tail of the waveguide and reduce the effective index of the waveguide, which results in stronger optical coupling into the resonator and improves phase matching with the resonator modes, respectively. However, the effective index of Si waveguides with widths that phase match well to tellurite's 2.08 material index are strongly width-dependent. This dependence can result in significant effective index variations of \pm 0.045 for a nominally $0.3-\mu \mathrm{m}$-wide waveguide assuming a $\pm 10 \mathrm{~nm}$ fabrication tolerance, which can lead to noticeable chip-to-chip and device-to-device variability during characterization. We used a $0.30-\mu \mathrm{m}$-wide Si bus waveguide around the cavity, which corresponds to an effective index of around 1.85. Figure 4(b) shows the calculated effective index of the resonator versus $\mathrm{TeO}_{2}$ film thickness, and for 40-, 100-, and 200- $\mu \mathrm{m}$ bending radii. The effective index of the resonator increases for thicker $\mathrm{TeO}_{2}$ films because the resonant mode becomes more confined in the $\mathrm{TeO}_{2}$ layer, from $\sim 1.80$ for a 500 -nm-thick coating up to $\sim 1.95$ for a 1100 -nm-thick $\mathrm{TeO}_{2}$ coating, which is in a range reasonably well phase matched to the chosen Si bus waveguide design. In a typical Si strip waveguide, the bending radius has minimal effect on its mode properties, except at extreme bending radii. However, in this microtrench cavity structure, the magnitude of the bend determines how strongly light is confined into the bottom corner of the cavity. The optical modes of devices with larger bending radii expand into the center of the microtrench structure, as can be seen by the large difference in effective mode areas for devices with different bending radii in Fig. 4(c). Expanding the mode inward toward the center of the cavity can potentially reduce scattering losses from interaction with the cavity sidewall, as shown previously, ${ }^{24}$ but larger mode areas can reduce the efficiency of nonlinear and rare-earth-doped active devices. In future designs, a thinner trench width, such as $2 \mu \mathrm{m}$, could be used to limit the expansion of the mode for larger radii devices, but risks introducing the opposite trench sidewall as a source of loss in 
the cavity. In general, optical confinement within the $\mathrm{TeO}_{2}$ film is large for all cavity designs, as seen in Fig. 4(d), and increases with $\mathrm{TeO}_{2}$ film thickness.

In addition to phase matching the effective index of the silicon bus waveguide to the tellurite glass resonator, it is also important to appropriately design the gap and length of the bus-resonator coupler to tune the coupling coefficient to the desired value for specific applications. We estimated the external/coupling $Q$ factors that can be expected between the bus waveguide and resonator using a coupled-mode theory calculation, in which the two-dimensional $X Y$ optical modes calculated for the silicon bus waveguide and the fundamental mode of the tellurite resonator by eigenmode simulations, as shown in Fig. 2(b), were overlapped with each other. The overlap integral of the modes then depends on the relative positions of the bus waveguide and resonator in the geometric positions of the coupler. We used the overlap integrals, relative phase of the two waveguides, and refractive index profile of the structure to calculate the transfer of optical power between the two waveguides for an initial light pulse travelling along the $Z$ direction of the bus waveguide. Further details on the theory and equations behind the model can be found in Refs. $50-52$. We carried out the simulation for a $0.3-\mu \mathrm{m}$-wide by $0.22-\mu \mathrm{m}$-thick silicon waveguide, with an effective waveguide index of 1.821, coupled to resonators with 500-, 700-, 900-, and 1100-nm-thick $\mathrm{TeO}_{2}$ coatings with effective waveguide indices of 1.838, 1.922, 1.969, and 1.999 , respectively, at coupling gaps of 0.1 to $0.45 \mu \mathrm{m}$. For simplicity, we assumed that the bottom of the resonator and waveguide are aligned such that there is no overetch of the oxide into BOX, and the coupling gap does not take into account any lateral offset of the oxide etch wall, such that the gap is referenced to the bottom right corner of the resonator cavity. Each simulation was run in steps of $0.5 \mu \mathrm{m}$ over a $120-\mu \mathrm{m}$ distance to ensure that at least one periodic beat of power from the bus waveguide to resonator and back to the waveguide occurs. Although the designed coupler is $104 \mu \mathrm{m}$ in length, additional coupling occurs in the transition section of the coupler and the various uncertainties in the device dimensions affect where on the sinusoidal coupling curve the measured value lies for a given wavelength. Therefore, we simply considered the peak calculated power that was coupled into the resonator over the simulated range as the maximum possible coupling coefficient for the geometry under consideration. The maximum simulated coupling coefficients were then converted into the minimum attainable external/ coupling $Q$ factor $^{53}$ for each device geometry and the results are plotted in Fig. 5.

The results demonstrate that for each of the waveguide dimensions studied the minimum attainable coupling $Q$ factor is around $0.2 \times 10^{5}$. The measured coupling $Q$ factors of devices would be expected to fall at or above these values depending on which phase of the sinusoidal coupling relationship they fall within. In general, each curve demonstrates a peak in coupling strength at a particular coupling gap, with significantly reduced coupling efficiency for relatively small variations in gap. Although it is expected to see reduced coupling efficiency at larger gaps, it is unusual for the coupling efficiency to also decrease at smaller gaps, as observed here. This is potentially a result of the phase difference between devices, as well as the vertical offset between

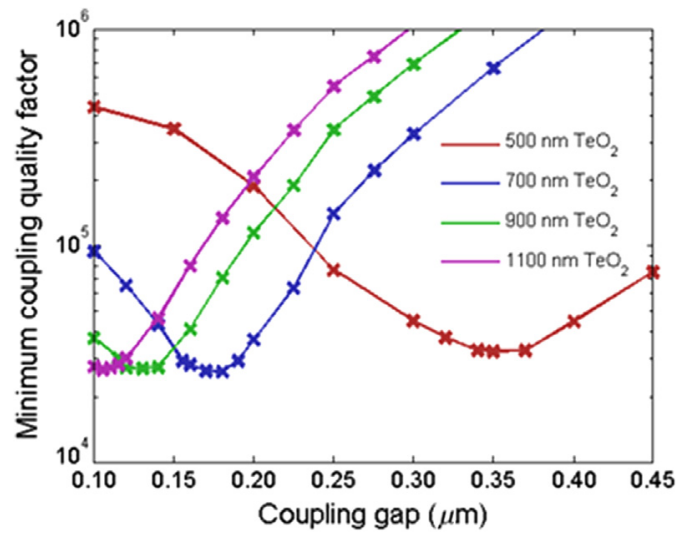

Fig. 5 Minimum attainable simulated external $Q$ factor for resonators coated in 500-, 700-, 900-, and $1100-\mathrm{nm}$-thick $\mathrm{TeO}_{2}$ coatings versus coupling gap distance to a $0.3-\mu \mathrm{m}$ wide by $0.22-\mu \mathrm{m}$ thick silicon bus waveguide. 
Frankis, Bonneville and Bradley: Tellurite glass microcavity resonators integrated on a silicon photonics...

the peak electric field of the bus waveguide and resonators' mode. For application of microcavities in lasers, high coupling $Q$ factors, typically of $\sim 1 \times 10^{6}$ and greater, are generally of interest to achieve roundtrip optical gain, which would require coupling gaps of $0.4 \mu \mathrm{m}$ and above based on these results.

\section{Microresonator Characterization}

To experimentally characterize the optical properties of the micro-trench resonators, we prepared three chips, coated in 495-, 775-, and 1115-nm-thick $\mathrm{TeO}_{2}$ films, in separate deposition processes. In each deposition, a silicon wafer witness piece used to measure film thickness via ellipsometry and an unpatterned thermally oxidized wafer used to measure film loss via the prism coupling method were mounted on the substrate holder along with the chip. We measured each $\mathrm{TeO}_{2}$ coating to have a film loss of less than $0.3 \mathrm{~dB} / \mathrm{cm}$ at $1550 \mathrm{~nm}$, suggesting high opticalquality thin films with low absorption and smooth surfaces and corresponding to material loss limited $Q$ factors of the devices of $>1 \times 10^{6}$. We measured the transmission of the microcavity devices using a fiber-chip edge coupling setup. TE polarized light from a 1550-nm tunable laser was launched into the chip via a polarization controller and cleaved fiber, and the transmitted light across the chip was coupled to another cleaved fiber and measured using a photodiode. Once aligned, the wavelength of the tunable laser was swept to characterize the transmission spectra of the devices. Figure 6(a) shows the measured resonance spectrum of a device with a 1115 -nm-thick $\mathrm{TeO}_{2}$ coating, and a nominal $0.2-\mu \mathrm{m}$ bus to resonator coupling gap, where two resonance modes can be observed across the range of the laser from 1510 to $1640 \mathrm{~nm}$, with a maximum extinction ratio of $9.1 \mathrm{~dB}$ and a measured coupling $Q$ factor of $1.1 \times 10^{5}$, for the fundamental mode at a wavelength of $1588 \mathrm{~nm}$. We fit the fundamental resonant modes of the device using coupled mode theory and a Lorentzian line shape to characterize the $Q$ factor of the device, as shown in Fig. 6(b), to have a maximum intrinsic $Q$ factor of $0.9 \times 10^{5}$, corresponding to a $4-\mathrm{dB} / \mathrm{cm}$ waveguide propagation loss.

The three initial $\mathrm{TeO}_{2}$ microcavity devices were characterized in the same method and the results are summarized in the first three entries of Table 1, showing significantly lower $Q$ factors for the thinner 495- and 775-nm-thick $\mathrm{TeO}_{2}$ coatings. Based on the low measured optical propagation losses of the films and minimal theoretical radiation losses based on the simulation results demonstrated in Fig. 3, it is assumed that the dominant source of loss in the resonator is scattering from surface roughness along the bottom and sidewalls of the microtrench cavity introduced during the oxide etch step. The optical modes of microcavities with thicker $\mathrm{TeO}_{2}$ films are more strongly confined within the $\mathrm{TeO}_{2}$ layer, leading to less scattering at the material interfaces and

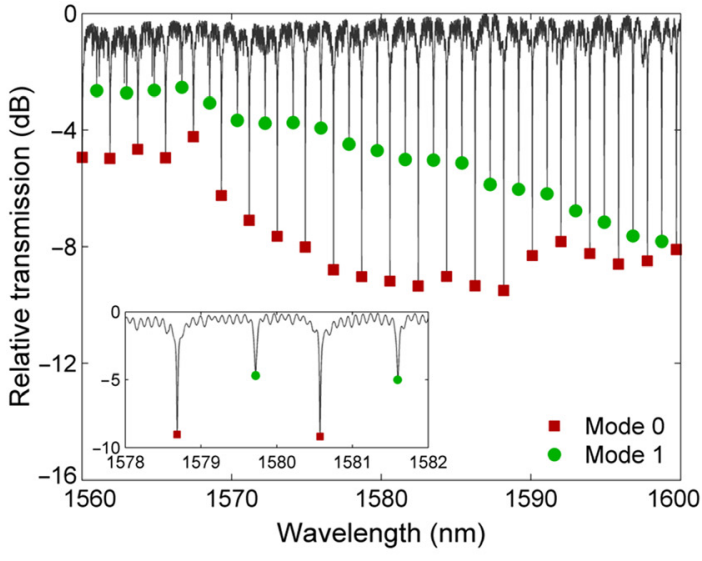

(a)

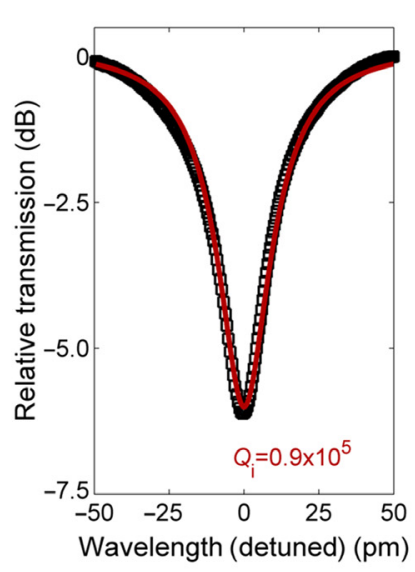

(b)

Fig. 6 (a) Transmission spectrum of a microtrench resonator with a 1115-nm-thick $\mathrm{TeO}_{2}$ coating, with inset showing a close-up view of two resonant free spectral ranges. (b) Fundamental resonance mode, fit with coupled mode theory to extract intrinsic $Q$ factors of $0.9 \times 10^{5}$ at a $1627-\mathrm{nm}$ wavelength. 
Table 1 Measured properties of optical microcavities with different Cytop and $\mathrm{TeO}_{2}$ coating thicknesses.

\begin{tabular}{ccccccc}
\hline \hline $\begin{array}{c}\text { Cytop } \\
\text { coating } \\
\begin{array}{c}\text { thickness } \\
(\mathrm{nm})\end{array}\end{array}$ & $\begin{array}{c}\mathrm{TeO}_{2} \\
\text { coating } \\
\text { thickness } \\
(\mathrm{nm})\end{array}$ & $\begin{array}{c}\mathrm{TeO}_{2} \\
\text { film loss } \\
(\mathrm{dB} / \mathrm{cm})\end{array}$ & $\begin{array}{c}\text { Maximum } \\
\text { extinction } \\
\text { ratio }(\mathrm{dB})\end{array}$ & $\begin{array}{c}\text { Minimum } \\
\text { external } \\
Q \text { factor }\end{array}$ & $\begin{array}{c}\text { Maximum } \\
\text { intrinsic } \\
Q \text { factor }\end{array}$ & $\begin{array}{c}\text { Propagation } \\
\text { loss } \\
(\mathrm{dB} / \mathrm{cm})\end{array}$ \\
\hline 0 & 495 & $0.1 \pm 0.1$ & 4.8 & $1.2 \times 10^{5}$ & $0.2 \times 10^{5}$ & 18.0 \\
0 & 775 & $0.3 \pm 0.2$ & 16.1 & $0.4 \times 10^{5}$ & $0.3 \times 10^{5}$ & 12.0 \\
0 & 1115 & $0.2 \pm 0.2$ & 8.9 & $1.1 \times 10^{5}$ & $0.9 \times 10^{5}$ & 4.0 \\
100 & 335 & $0.3 \pm 0.2$ & 4.8 & $3.3 \times 10^{5}$ & $1.2 \times 10^{5}$ & 3.0 \\
100 & 630 & $0.2 \pm 0.2$ & 2.5 & $4.8 \times 10^{5}$ & $2.1 \times 10^{5}$ & 1.7 \\
100 & 840 & $0.1 \pm 0.1$ & 5.0 & $1.7 \times 10^{5}$ & $1.5 \times 10^{5}$ & 2.4 \\
50 & 970 & $0.4 \pm 0.2$ & 3.5 & $2.0 \times 10^{5}$ & $1.8 \times 10^{5}$ & 2.0 \\
\hline \hline
\end{tabular}

the higher observed $Q$ factors, but are still below the values needed for efficient optically pumped light-emitting microcavity devices. In regards to coupling $Q$ factors, the measured values are all larger than the simulated minimum values seen in Fig. 5. This is largely due to the increased coupling gap that will occur due to the lateral offset created by the sloped oxide etch wall and overetching of devices into the BOX, as well as the pulley coupler design not optimizing toward the maximum coupling coefficient. However, in the case of the 775-nm-thick film, we are able to see a minimum external $Q$ factor of $0.4 \times 10^{5}$, approaching the minimum values predicted in simulations and critical coupling.

To reduce the roughness of the trench interface, we investigated coating chips in a thin fluoropolymer (Cytop) layer, using a spin-on and low temperature $\left(180^{\circ} \mathrm{C}\right)$ baking technique before depositing the $\mathrm{TeO}_{2}$ coating. In general, this technique demonstrates a consistent improvement in device $Q$ factors, with all devices measuring $>10^{5}$ for film thicknesses from 335 to $970 \mathrm{~nm}$ as seen in the last 4 entries of Table 1. Figure 7(a) shows the transmission spectrum for a device with a 100-nm-thick Cytop coating and 630-nm-thick $\mathrm{TeO}_{2}$ film, which displays three resonant modes. We again fit the fundamental mode using coupled mode theory, as shown in Fig. 7(b), to extract an internal $Q$ factor of $2.1 \times 10^{5}$, which corresponds to a waveguide loss of $1.7 \mathrm{~dB} / \mathrm{cm}$ within the resonator and approaches the values needed to demonstrate efficient nonlinear

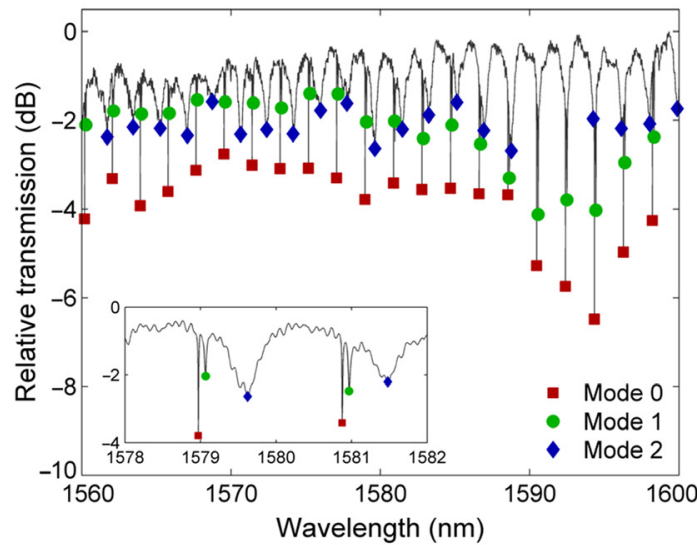

(a)

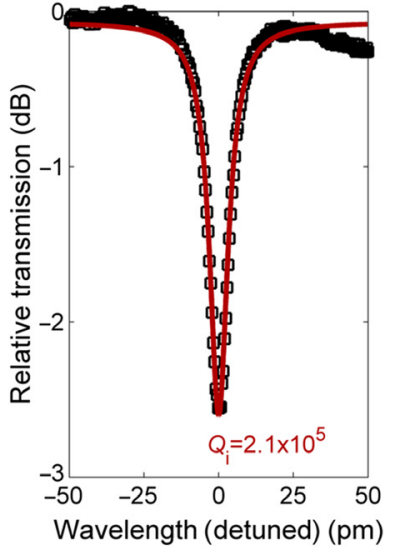

(b)

Fig. 7 (a) Transmission spectrum of microtrench cavity with a 100-nm-thick Cytop layer under a 630-nm-thick $\mathrm{TeO}_{2}$ coating, with inset showing a close-up view of two resonant free spectral ranges. (b) Fundamental resonant mode fitted using coupled mode theory to extract an intrinsic $Q$ factor of $2.1 \times 10^{5}$ at a $1566-\mathrm{nm}$ wavelength. 
functionality $\left(1 \times 10^{6}\right)$ and net rare-earth roundtrip gain and lasing $\left(5 \times 10^{5}\right) .{ }^{25}$ Further reduction of the microtrench surface roughness, which can be accomplished by an improved $\mathrm{SiO}_{2}$ trench etch step within the foundry and hydrofluoric acid (HF) etching the trench before deposition, ${ }^{31}$ can potentially lead to even higher $Q$ factors in $\mathrm{TeO}_{2}$ microtrench cavities. As seen in the results of Table 1, the Cytop-coated samples have significantly larger coupling $Q$ factors, likely as a result of the coating creating a further lateral offset between the waveguide and tellurite resonator.

\section{Conclusions}

We have demonstrated integrated $\mathrm{TeO}_{2}$ microtrench resonators on silicon photonic chips using a standard wafer-scale foundry process and a straightforward low-temperature post-processing $\mathrm{TeO}_{2}$ deposition step. Calculations show that even with $\mathrm{TeO}_{2}$ coating thicknesses of over $1000 \mathrm{~nm}$, the microcavities can reasonably be bent below a $40-\mu \mathrm{m}$ radius, which maintains a compact footprint on scale with silicon photonic devices. Measurements show that a 1115-nmthick $\mathrm{TeO}_{2}$ film deposited onto a device as received from the foundry yields an internal $Q$ factor of $0.9 \times 10^{5}$. Using a fluoropolymer coating to smooth the cavity is shown to enable a device with a $630-\mathrm{nm} \mathrm{TeO}_{2}$ coating to reach a $Q$ factor of $2.1 \times 10^{5}$. Further investigations into smoothing the microtrench interface, such as HF etching of the oxide, and reducing the microtrench etch roughness could allow for even higher $Q$ factors and the design and fabrication of efficient optically pumped nonlinear optical and rare-earth-doped devices. These results represent a potential pathway toward monolithic fabrication of low cost and high performance nonlinear and rareearth-doped active tellurite glass devices in silicon photonic platforms, with applications in frequency comb generation, environmental and biological sensing, and communications.

\section{Acknowledgments}

We acknowledge the Center for Emerging Device Technologies (CEDT) and Shahram GhanadTavakoli and Doris Stevanovic for use of and assistance with the sputtering system, Zhilin Peng and John Luxat of the Centre for Advanced Nuclear Systems (CANS) Post Irradiation Examination Hot Cell Facility for use of the FIB/SEM and the CMC Microsystems SiEPIC program for organizing the fabrication tape out. This work was supported by the Natural Sciences and Engineering Research Council of Canada (Grant Nos. STPGP 494306 and RGPIN-201706423); Canadian Foundation for Innovation (CFI Project \# 35548); and Ontario Research Fund (ORF Project \# 35548).

\section{References}

1. W. Bogaerts et al., "Nanophotonic waveguides in silicon-on-insulator fabricated with CMOS technology," J. Lightwave Technol. 23(1), 401-412 (2005).

2. W. Bogaerts et al., "Silicon microring resonators," Laser Photonics Rev. 6(1), 47-73 (2012).

3. P. Dong et al., "Silicon photonic devices and integrated circuits," Nanophotonics 3(4-5), 215-228 (2014).

4. W. Bogaerts and L. Chrostowski, "Silicon photonics circuit design: methods, tools and challenges," Laser Photonics Rev. 12(4), 1700237 (2018).

5. J. Leuthold, C. Koos, and W. Freude, "Nonlinear silicon photonics," Nat. Photonics 4(8), 535-544 (2010).

6. A. D. Bristow, N. Rotenberg, and H. M. Van Driel, "Two-photon absorption and Kerr coefficients of silicon for 850-2200 nm," Appl. Phys. Lett. 90(19), 191104 (2007).

7. A. J. Kenyon, "Recent developments in rare-earth doped materials for optoelectronics," Prog. Quantum Electron. 26(4-5), 225-284 (2002).

8. A. J. Kenyon, "Erbium in silicon," Semicond. Sci. Technol. 20(12), R65 (2005).

9. P. Del'Haye et al., "Optical frequency comb generation from a monolithic microresonator," Nature 450(7173), 1214-1217 (2007). 
Frankis, Bonneville and Bradley: Tellurite glass microcavity resonators integrated on a silicon photonics...

10. I. H. Agha, Y. Okawachi, and A. L. Gaeta, "Theoretical and experimental investigation of broadband cascaded four-wave mixing in high-Q microspheres," Opt. Express 17(18), 16209-16215 (2009).

11. S. M. Spillane, T. J. Kippenberg, and K. J. Vahala, "Ultralow-threshold Raman laser using a spherical dielectric microcavity," Nature 415(6872), 621-623 (2002).

12. T. J. Kippenberg et al., "Ultralow-threshold microcavity Raman laser on a microelectronic chip," Opt. Lett. 29(11), 1224-1226 (2004).

13. L. Yang, D. K. Armani, and K. J. Vahala, "Fiber-coupled erbium microlasers on a chip," Appl. Phys. Lett. 83(5), 825-826 (2003).

14. J. Kalkman et al., "Fabrication and characterization of erbium-doped toroidal microcavity lasers," J. Appl. Phys. 99(8), 083103 (2006).

15. Y. Yang et al., "Tunable erbium-doped microbubble laser fabricated by sol-gel coating," Opt. Express 25(2), 1308-1313 (2017).

16. D. W. Vernooy et al., "High-Q measurements of fused-silica microspheres in the near infrared," Opt. Lett. 23(4), 247-249 (1998).

17. S. Berneschi et al., "High Q silica microbubble resonators fabricated by arc discharge," Opt. Lett. 36(17), 3521-3523 (2011).

18. D. K. Armani et al., "Ultra-high-Q toroid microcavity on a chip," Nature 421(6926), 925-928 (2003).

19. D. Armani et al., "Electrical thermo-optic tuning of ultrahigh-Q microtoroid resonators," Appl. Phys. Lett. 85(22), 5439-5441 (2004).

20. A. Kovach et al., "Optically tunable microresonator using an azobenzene monolayer," AIP $A d v$. 10(4), 045117 (2020).

21. J. Richter, M. P. Nezhad, and J. Witzens, "Monolithically integrated waveguide-coupled silica microtoroids," in Opto-Electron. and Commun. Conf., pp. 1-3 (2005).

22. J. Richter et al., "High-Q inverted silica microtoroid resonators monolithically integrated into a silicon photonics platform," Opt. Express 26(21), 27418-27440 (2018).

23. K. Y. Yang et al., "Ultra-high-Q silica-on-silicon ridge-ring-resonator with an integrated silicon nitride waveguide," in CLEO: Sci. and Innov., OSA Technical Digest Series, Optical Society of America, Paper JTh4B.7 (2016).

24. Z. Su et al., "High-Q-factor $\mathrm{Al}_{2} \mathrm{O}_{3}$ micro-trench cavities integrated with silicon nitride waveguides on silicon," Opt. Express 26(9), 11161-11170 (2018).

25. J. D. B. Bradley et al., "Monolithic erbium-and ytterbium-doped microring lasers on silicon chips," Opt. Express 22(10), 12226-12237 (2014).

26. Z. Su et al., "Ultra-compact and low-threshold thulium microcavity laser monolithically integrated on silicon," Opt. Lett. 41(24), 5708-5711 (2016).

27. H. C. Frankis et al., "Four-wave mixing in a high-Q aluminum oxide microcavity on silicon," in CLEO: Sci. and Innov., OSA Technical Digest Series, Optical Society of America, Paper STh3I.3 (2018).

28. M. Xin et al., "Optical frequency synthesizer with an integrated erbium tunable laser," Light Sci. Appl. 8(1), 1-8 (2019).

29. J. Notaros et al., "CMOS-Compatible optical phased array powered by a monolithicallyintegrated erbium laser," J. Lightwave Technol. 37(24), 5982-5987 (2019).

30. N. Li et al., "A silicon photonic data link with a monolithic erbium-doped laser," Sci. Rep. 10(1), 1114 (2020).

31. P. Jean et al., "Etchless chalcogenide microresonators monolithically coupled to silicon photonic waveguides," Opt. Lett. 45(10), 2830-2833 (2020).

32. K. Tanaka, "Photoinduced processes in chalcogenide glasses," Curr. Opin. Solid State Mater. Sci. 1(4), 567-571 (1996).

33. K. Tanaka, "Photoinduced structural changes in amorphous semiconductors," Semiconductors 32(8), 861-866 (1998).

34. X. Gai et al., "Progress in optical waveguides fabricated from chalcogenide glasses," Opt. Express 18(25), 26635-26646 (2010).

35. S. J. Madden and K. T. Vu, "Very low loss reactively ion etched tellurium dioxide planar rib waveguides for linear and non-linear optics," Opt. Express 17(20), 17645-17651 (2009). 
Frankis, Bonneville and Bradley: Tellurite glass microcavity resonators integrated on a silicon photonics...

36. H. C. Frankis et al., "Low-loss $\mathrm{TeO}_{2}$-coated $\mathrm{Si}_{3} \mathrm{~N}_{4}$ waveguides for application in photonic integrated circuits," Opt. Express 27(9), 12529-12540 (2019).

37. S. H. Kim, T. Yoko, and S. Sakka, "Linear and nonlinear optical properties of $\mathrm{TeO}_{2}$ glass," J. Am. Ceram. Soc. 76(10), 2486-2490 (1993).

38. H. M. Mbonde, H. C. Frankis, and J. D. B. Bradley, "Enhanced nonlinearity and engineered anomalous dispersion in $\mathrm{TeO}_{2}$-coated $\mathrm{Si}_{3} \mathrm{~N}_{4}$ waveguides," IEEE Photonics J. 12(2), 1-10 (2020).

39. R. Stegeman et al., "Tellurite glasses with peak absolute Raman gain coefficients up to 30 times that of fused silica," Opt. Lett. 28(13), 1126-1128 (2003).

40. K. Vu and S. Madden, "Tellurium dioxide erbium doped planar rib waveguide amplifiers with net gain and $2.8 \mathrm{~dB} / \mathrm{cm}$ internal gain," Opt. Express 18(18), 19192-19200 (2010).

41. K. Vu, S. Farahani, and S. Madden, "980nm pumped erbium doped tellurium oxide planar rib waveguide laser and amplifier with gain in S, C and L band," Opt. Express 23(2), 747-755 (2015).

42. H. C. Frankis et al., "Erbium-doped $\mathrm{TeO}_{2}$-coated $\mathrm{Si}_{3} \mathrm{~N}_{4}$ waveguide amplifiers with $5 \mathrm{~dB}$ net gain," Photonics Res. 8(2), 127-134 (2020).

43. K. M. Kiani et al., "Thulium-doped tellurium oxide waveguide amplifier with $7.6 \mathrm{~dB}$ net gain on a silicon nitride chip," Opt. Lett. 44(23), 5788-5791 (2019).

44. H. C. Frankis et al., "A tellurium oxide microcavity resonator sensor integrated on-chip with a silicon waveguide," Sensors 18(11), 4061 (2018).

45. T. J. Kippenberg, R. Holzwarth, and S. A. Diddams, "Microresonator-based optical frequency combs," Science 332(6029), 555-559 (2011).

46. L. He et al., "Detecting single viruses and nanoparticles using whispering gallery microlasers," Nat. Nanotechnol. 6(7), 428-432 (2011).

47. N. Li et al., "A silicon photonic data link with a monolithic erbium-doped laser," Sci. Rep. 10(1), 1114 (2020).

48. C. I. Van Emmerik et al., "Single-layer active-passive $\mathrm{Al}_{2} \mathrm{O}_{3}$ photonic integration platform," Opt. Mater. Express 8(10), 3049-3054 (2018).

49. M. Soltani, S. Yegnanarayanan, and A. Adibi, "Ultra-high Q planar silicon microdisk resonators for chip-scale silicon photonics," Opt. Express 15(8), 4694-4704 (2007).

50. R. Stoffer, K. R. Hiremath, and M. Hammer, "Comparison of coupled mode theory and FDTD simulations of coupling between bent and straight optical waveguides," AIP Conf. Proc. 709(1), 366-377 (2004).

51. K. R. Hiremath and M. Hammer, "Modeling of 2D cylindrical integrated optical microresonators," Master's Report, University of Twente (2003).

52. W. P. Huang and J. Mu, "Complex coupled-mode theory for optical waveguides," Opt. Express 17(21), 19134-19152 (2009).

53. M. Soltani, "Novel integrated silicon nanophotonic structures using ultra-high Q resonators," PhD Dissertation, Georgia Institute of Technology (2009).

Biographies of the authors are not available. 